\title{
Filosofía y ciudadanía: entre afirmación y formación
}

\section{Maximiliano Durán}

Doctor en Filosofía

Universidad de Buenos Aires -

Argentina

Maximiliano1771@gmail.com

https://orcid.org/000o-0002-7213-6834

Fabiana Fernandes Ribeiro

\section{Martins}

Magíster en Educación

Colégio Pedro II, Rio de Janeiro - Brasil fabitins@gmail.com

https://orcid.org/0000-0003-1749-0547
Artículo de reflexión

Recepción: 15 de enero de 2019 Aprobación: 02 de marzo de 2019 https://doi.org/10.19053/22160159.v10.n23.2019.9730

\section{Resumen}

La formación ética y ciudadana se constituye como uno de los pilares fundamentales de las escuelas modernas latinoamericanas. Desde los inicios del siglo XIX, intelectuales y políticos ven en la institución escolar el medio adecuado de formación social y política de sus habitantes. Algunos autores asumen, desde diversas perspectivas, la escuela como una especie de máquina de ciudadanía. Según nuestro punto de vista, más allá de los cambios políticos e institucionales ocurridos en los últimos doscientos años, esta idea continúa vigente. La escuela entendida como máquina de ciudadanía supone una especie de distancia entre el ciudadano formado y aquel que aún no lo está. Dicha distancia se sostiene en una concepción de la igualdad como objetivo. De acuerdo con esta forma de pensar la ciudadanía, los niños no son iguales a los ciudadanos formados. En el mejor de los casos, la ciudadanía que afirma la escuela es, al menos en sus inicios, incompleta, inacabada y debe ser formada. La pregunta que inspira nuestro trabajo es: ¿quiénes son aquellos que llamamos ciudadanos, aquellos a los que nuestros esfuerzos pedagógicos están dirigidos?

Palabras claves: ciudadanía, igualdad, escuela 


\title{
Philosophy and citizenship: between affirmation and training
}

\begin{abstract}
Ethical and citizen education is one of the fundamental pillars of modern Latin American schools. Since the beginning of the nineteenth century, intellectuals and politicians have seen school as a special locus for the social and political formation of their inhabitants. Several authors defend, in different perspectives, that school is a type of citizenship machine. Even though political and institutional changes have taken place in the last two hundred years, we argue that this idea still remains valid. The school understood as a citizenship machine supposes a type of distance between the formed citizen and the one that is not yet formed. This distance is based on a conception of equality as an objective to be achieved. According to this way of conceiving citizenship, children are not equal to educated citizens. In other words, the citizenship which is affirmed by school is that one that has to be formed through the educational process. The question that inspires our work is: who are those we call citizens, those to whom our pedagogical efforts are directed?
\end{abstract}

Keywords: citizenship, equality, school

\section{La philosophie et la citoyenneté : entre l'affirmation et la formation}

\section{Résumé}

La formation éthique et citoyenne constitue l'un des piliers fondamentaux des écoles modernes latino-américaines. Depuis le début du XIXe siècle, les intellectuels et les hommes politiques considèrent les institutions scolaires comme le moyen approprié de formation sociale et politique de leurs habitants. Plusieurs auteurs perçoivent l'école, à partir de perspectives différentes, comme une sorte de machine de citoyenneté. Selon nous, au- 
delà des changements politiques et institutionnels intervenus au cours des deux cents dernières années, cette idée est toujours en vigueur. L'école entendue comme une machine de citoyenneté suppose une sorte de fossé entre les citoyens éduqués et ceux qui ne sont pas encore éduqués. Ce fossé est fondé sur une conception d'égalité en tant qu'objectif. Conformément à cette manière de concevoir la citoyenneté, les enfants ne sont pas égaux aux citoyens éduqués. Au mieux, la citoyenneté affirmée par l'école est, du moins en principe, incomplète, inachevée, et elle doit être éduquée. La question qui inspire ce travail est : qui sont ceux que l'on appelle des citoyens ? Estce qu'ils sont ceux pour lesquels nos efforts pédagogiques sont faits?

Mots-clés : citoyenneté, égalité, école

\section{Filosofia e cidadania: entre afirmação e formação}

\section{Resumo}

A formação ética e cidadã se constitui como um dos pilares fundamentais das escolas modernas latino-americanas. Desde o início do século XIX, intelectuais e politicos vêm na instituição escolar um meio adequado para a formação social e política de seus habitantes. Alguns autores defendem, a partir de perspectivas diversas, a escola como um tipo de máquina de cidadania. Segundo nosso ponto de vista, para além das mudanças politicas e institucionais ocorridas nos últimos duzentos anos, essa ideia continua vigente. A escola entendida como máquina de cidadania supõe um tipo de distância entre o cidadão formado e aquele que ainda não está formado. Essa distância está sustentada numa concepção de igualdade como objetivo a ser alcançado. De acordo com esse modo de pensar a cidadania, as crianças não são iguais aos cidadãos formados. No melhor dos casos, a cidadania que a escola afirma é, pelo menos no início, incompleta, inacabada e deve ser formada. A pergunta que inspira nosso trabalho é: quem são aqueles que chamamos de cidadãos, aqueles aos quais nossos esforços pedagógicos estão dirigidos?

Palavras-chave: cidadania, igualdade, escola 


\section{Introducción}

En el presente trabajo presentamos una forma distinta de pensar la ciudadanía y la relación que tiene la filosofía con ese concepto en la escuela primaria. Para lograr nuestro objetivo divimos el trabajo en tres partes. En la primera, realizamos un breve análisis histórico del origen del concepto de ciudadano en América. Para ello, nos apoyamos en el desarrollo histórico realizado por nosotros en un artículo publicado en Estudios de filosofía práctica e historia de las ideas (Durán, 2010). A partir de la reapropiación y relectura del mismo, nos proponemos mostrar que dicho concepto se constituye sobre la base de una idea de igualdad como objetivo. Esta concepción, en última instancia, afirma aquello que pretende negar: la desigualdad. En otras palabras, decimos que la supuesta igualdad sobre la que se constituye la ciudadanía que nuestras escuelas pretenden formar no existe. La segunda parte del texto presenta otra forma de entender la ciudadanía, a partir de un concepto de igualdad radicalmente novedoso pensado por Simón Rodríguez. De acuerdo con el pensamiento de este autor, la ciudadanía es la afirmación de un axioma igualitario que se despliega en la práctica escolar. La tercera parte del trabajo intenta relacionar el pensamiento de Rodríguez sobre la ciudadanía y la igualdad con la práctica cotidiana de la filosofía en la escuela primaria. Para ello nos apoyamos en el proyecto de filosofía Em Caxias a filosofía en-caixa? esarrollado por el Núcleo de Estudios de Filosofías e Infancias [NEFI ${ }^{1}$ en el Municipio de Caxias del Estado de Río de Janeiro en Brasil. Este proyecto, coordinado por Walter Kohan, asume la idea de ciudadanía de Rodríguez y afirma la igualdad como un principio del cual se parte para hacer filosofía. La igualdad en Caxias se encuentra en la base del hacer filosofía de nuestros niños y niñas. Así, la ciudadanía no es el resultado de un proceso, sino una declaración de la cual se parte.

De esta manera, al finalizar nuestro trabajo, pensamos contar con elementos para trabajar la ciudadanía en la escuela. Para nosotros, el pensamiento de Rodríguez es una inspiración. Inventamos o erramos. No hay un orden para imitar ni un modelo que copiar. Es preciso hacer la escuela del pensamiento, de la filosofía, de una vida que interrumpa las vidas que estamos viviendo

1 El NEFI es un grupo compuesto por una heterogeneidad de participantes, que incluyen estudiantes de escuela básica, graduados, post graduados, y becarios de postgraduación. Para saber más sobre el NEFI, visite: www.filoeduc.org 
y genere las condiciones para una nueva vida. En tiempos oscuros de la política en nuestros países, en que los aires conservadores parecen haber ganado nuevo impulso, resistir se ha tornado una necesidad para los que pensamos que las cosas siempre pueden ser de otra manera.

\section{Historia del ciudadano}

Las investigaciones recientes en el campo historiográfico muestran que la construcción de la ciudadanía en América del Sur durante los siglos XVIII y XIX no puede ser leída como un proceso lineal, en el que categorías propias del antiguo régimen fueron negadas, desechadas y reemplazadas completamente en pos de otras conceptualizaciones y clasificaciones acordes a las circunstancias políticas del periodo. En el caso puntual del concepto de ciudadano, es posible observar la incorporación paulatina de elementos modernos sobre la base de categorías tradicionales existentes a lo largo de todo el proceso de su construcción. En las próximas líneas intentaremos analizar este proceso durante el siglo XVIII y las primeras dos décadas del siglo XIX. El objetivo de esta primera parte del trabajo consiste en mostrar cómo el concepto de ciudadano que se gestó en América durante este periodo fue la expresión de una tensión conceptual entre categorías modernas y otras del antiguo régimen utilizadas para resolver problemas coyunturales vinculados a los intereses de ciertos grupos.

De acuerdo con los trabajos de Cansanello (2008), Núñez (2007) y Guerra (1999), el concepto de ciudadano en América Latina tiene una larga tradición. De acuerdo con estos autores, dicho concepto presenta una evolución en la que los elementos modernos van surgiendo paulatinamente sobre elementos previos propios del antiguo régimen a la modernidad. Podría decirse que el ciudadano moderno, tal como nosotros lo concebimos, es el resultado de un proceso histórico cuyos inicios habrán de buscarse en conceptos antiguos, tal como el de vecino.

La vecindad se encuentra presente en la definición de ciudadano propuesta por el Diccionario de Autoridades de 1725. De acuerdo con este texto, el ciudadano, es el vecino de una ciudad que goza de sus privilegios y está obligado a sus cargas. Se trata de una categoría político-social de la colonia sujeta a los derechos 
específicos de cada una de las comunidades que, a diferencia de la abstracción propia del ciudadano moderno, se refiere a un hombre concreto y territorializado dentro de una comunidad determinada. Este par vecino-ciudadano se mantuvo hasta el la primera década del siglo XIX. La Carta Gaditana de 1812 comienza a postular un concepto de ciudadanía más próximo a la nación que a la ciudad. Allí comienza a hablarse de derechos y obligaciones en lugar de cargas y privilegios. Quijada (2008) sostiene que la Carta Gaditana se valió de los términos de nación y ciudadanía, en el sentido moderno, para cohesionar a la metrópoli y a los territorios de ultramar dentro de una misma estructura política homogénea. La noción de ciudadanía no reconocía distinciones fundadas en el estatuto personal o colectivo. La condición de ciudadano igualaba a las personas. Esta nueva categoría ignoraba los privilegios personales, estamentarios o aquellos derivados del lugar de residencia y expresaba una igualdad y universalidad sin precedentes en el territorio americano (Durán, 2010).

Ahora bien, esta igualdad (Cansanello, 2008; Guerra, 1999; Núñez, 2007) posee una larga historia que puede remontarse a la tradición pactista española. De la misma forma que el par vecinociudadano, es posible hablar de una par libertad medieval-libertad moderna sobre la que se constituye el concepto de ciudadano. Según nuestro punto de vista, la igualdad implícita en el concepto de ciudadano tiene sus bases en el pensamiento de los padres Suárez y de Vitoria (Durán, 2010). Este último es una referencia indiscutida a la hora de tematizar la igualdad en el continente americano. En sus Reelecciones sobre los indios y el derecho de guerra (1946), hace referencia a la existencia de derechos que ninguna autoridad podía ignorar. Estos, a diferencia de los modernos, se fundan en la naturaleza del ser del hombre y no en su voluntad. Siguiendo a Tomás de Aquino, sostienen que, dada su capacidad racional, los hombres participan a través de la Ley Natural de la Ley Eterna, impuesta por Dios (Tomas de Aquino, 1947, I-II, q. 91, a. 2). De acuerdo con ello, se sostiene la existencia de una suerte de plano de igualdad entre los hombres, en la medida en que todos poseen la capacidad de actuar como es debido de acuerdo a la Ley Natural, en virtud de su forma racional. Como puede observarse, la igualdad que se sostiene en este punto es de carácter religioso, en la medida que ella es posibilidad, producto y reflejo de la potestad divina. 
Según Donghi (2010), esta concepción de igualdad pensada por el humanismo español es utilizada para establecer límites al derecho temporal y fijar condiciones a la vida civil. De acuerdo con ella, la igualdad reside en la condición religiosa del hombre y en virtud de ella debe ser respetada. Nada establece sobre la transformación de las condiciones políticas y sociales de los hombres. Con el correr del tiempo, el ius-naturalismo moderno abandona paulatinamente esta concepción religiosa de la igualdad para apoyarla en la razón. Para ellos habría una supuesta igualdad original, en la que no hay amos, ni esclavos, ni ricos, ni pobres, que es quebrada con el estado de guerra propio del poder arbitrario y del interés egoísta (Durán, 2010). La dimensión civil de la igualdad, entendida como la igualdad de derechos para todos, es concebida entonces como una suerte de restitución parcial de esa igualdad originaria implícita en el derecho natural. Ambas dimensiones sostienen que la desigualdad no es un rasgo propio del hombre y asumen que, en una situación ideal, esto debe ser corregido. Tanto para el humanismo español, como para el ius-naturalismo moderno de autores como Rousseau, Hobbes y Locke, la igualdad es algo que debe ser restituido en el estado de situación actual (Donghi, 2010). En otras palabras, sostienen que lo que existe es la desigualdad y que la igualdad es un elemento ideal a conseguir.

Sobre esta base de igualdad a ser alcanzada se constituye un concepto de ciudadanía en América con las mismas características ideales que la igualdad propuesta por ambos, el ius-naturalismo. Es decir, la ciudadanía en América tras las guerras de Independencia es una categoría declamada idealmentey ausente en la realidad cotidiana. En el mejor de los casos, se tratará de conseguir paulatinamente a través de diversos procesos políticos y sociales, tanto una como otra. De esta manera pensamos que es posible sostener que tanto la ciudadanía como la igualdad en América se plantean como objetivos a ser alcanzados a lo largo de su historia. En la próxima sección del texto presentaremos el pensamiento de Simón Rodríguez como una forma original y novedosa de pensar ambos conceptos.

\section{Ciudadano de Rodríguez}

El concepto de ciudadano, pensado por Simón Rodríguez se distingue del resto de los pensadores de su época por una especial y 
particular forma de entender la igualdad y la universalidad (Durán, 2010). Rodríguez tiene una forma original de entender ambos conceptos. Existe una interrelación entre universalidad e igualdad anclada en el gesto de la afirmación y el ofrecimiento. A diferencia de la tradición, que considera tanto uno como otro en tanto objetivo y propiedad, valor o ley, Rodríguez los asume en tanto afirmación y ofrecimiento. La igualdad que piensa y actúa el maestro, lejos de ser un horizonte a conseguir en un tiempo incierto, es una realidad que se afirma y se practica en el aquí y ahora. A su vez la universalidad no es pensada como una relación entre una totalidad y un valor que rige sobre esa totalidad y cada uno de sus elementos, ni como una propiedad por todos compartida, sino como el encabezado de un anuncio de un nuevo presente para todos. Desde nuestra perspectiva, la ciudadanía se constituye en el pensamiento de Rodríguez a partir de la materialización de estos dos conceptos en una práctica concreta que transforma el estado de cosas reinante tras las guerras de independencia.

Entre 1825 y 1826, Bolívar piensa un proyecto constitucional para la nueva República de Bolívar, donde piensa la ciudadanía a partir de su condición letrada. En otras palabras, solo sería considerado para la ciudadanía aquel que supiera leer y escribir. Con tal propósito, Rodríguez fue convocado para pensar un proyecto de educación popular. El maestro debía establecer las bases y condiciones para que la ciudadanía pudiese establecerse en la nueva República. Para los sectores acomodados y tradicionales de la ciudad, la tarea encomendada a Rodríguez asumía la igualdad y la ciudadanía como una ausencia ideal que, en el mejor de los casos, debía construirse. Sin embargo, el accionar de Rodríguez fue muy distinto del deseado por las élites. Abrió una escuela en la que todos los niños y niñas de todas las castas pudiesen asistir en calidad de iguales. La escuela de Chuquisaca se caracterizó porque en ella los niños no iban a tornarse ciudadanos, sino porque ya lo eran. Esta idea se apoya en una afirmación que realiza en Sociedades Americanas. En este texto pionero, en el que recoge muchas de las ideas que se propuso implementar en Bolivia, escribe: "escuelas para todos porque todos son ciudadanos" (Rodríguez, 1999a, p. 284). Absolutamente todos y todas ingresaban en la escuela porque eran iguales. Esta decisión del maestro caraqueño supuso la puesta en práctica de un principio igualitario sin precedentes en 
el continente. La igualdad en la ciudadanía afirmada por Rodríguez tiene un carácter axiomático. Ella está en el inicio y base de su proyecto político-educativo y no al final, como establece el resto de los proyectos educativos. Ella se expresa y desarrolla en una práctica fundada en el ingreso y recepción de todos los niños y niñas de la ciudad sin establecer diferenciaciones de sexo, etnia, posición económica y religión.

La afirmación igualitaria implícita en esta decisión se encuentra en relación directa con la universalidad, tal como la entiende el maestro en el inicio de la frase. El cuantificador universal con el que inicia la oración, lo entendemos como el anuncio de una novedad que se dirige a todos. Quiere decir que, independientemente de la condición de indio, criollo, cholo, negro, zambo, mulato o peninsular, una persona va a la escuela porque es ciudadana. Y es ciudadana porque todas ellas son iguales. Esta condición igualitaria anclada y ofrecida universalmente es el elemento central sobre el que Rodríguez constituye el concepto de ciudadanía. Podría decirse que la ciudadanía es entendida como el ofrecimiento incondicionado de una afirmación igualitaria que inaugura un nuevo presente. Es la invitación irrestricta a la construcción de una realidad potente y original en la cual la igualdad no precisa ser demostrada, sino tan solo practicada. La originalidad de esta propuesta no reside en la referencia a elegir y ser elegido que tienen todos los individuos en el interior de los sistemas de república, sino en un sentido mucho más profundo. Para nosotros, la ciudadanía en Rodríguez expresa una forma diferente y nueva de vida, cuyo principio estructurante, organizador y operativo es la igualdad. En otras palabras, pensamos que ciudadanos, en el pensamiento de Rodríguez, es el nombre de aquellos que deciden vivir como iguales. Este principio, esta forma novedosa de pensar la igualdad es una inspiración, para el trabajo de filosofía con niños y niñas en el proyecto desarrollado en Duque de Caxias a las afueras de la ciudad de Río de Janeiro. A continuación explicamos la forma en la que materializamos dicha inspiración.

\section{Ciudadano de la escuela}

Desde 2007, el NEFI de la Universidad del Estado de Río de Janeiro, lleva adelante un programa de filosofía en dos escuelas primarias 
municipales en la ciudad de Duque de Caxias. El título del proyecto en portugués es Em Caxias a filosofía en-caixa?. El título mismo juega con los sentidos de las palabras de Em Caxias - nombre del municipio-y en-caixa -encaja-y podría ser traducido como ¿ $L a$ filosofía en Caxias encaja?. Dicho programa se propone contribuir al mejoramiento de la enseñanza en la escuela pública a través de prácticas filosóficas con niños, jóvenes y adultos. Aborda y despliega las posibilidades de la filosofía como experiencia de pensamiento en distintos niveles del sistema de educación pública -educación infantil, fundamental y jóvenes y adultos-. Propone un trabajo constante en la formación de profesores a partir de experiencias filosóficas. Estas asumen que la filosofía no es un discurso que se impone a otros en dónde se encuentra la verdad y cuál es la forma de encontrarla, enseñarla y aprenderla. Para nosotros, se trata siempre de un ejercicio de transformación de nosotros mismos. En este sentido, la pregunta "a filosofia en-caixa?" no es un mero juego de palabras: se trata de vivenciar la filosofía como una caja de herramientas (Deleuze \& Foucault, 2006).

A través de encuentros periódicos en la universidad y en las escuelas, el programa intenta explorar las posibilidades educativas de la filosofía con niños, jóvenes y adultos. En este punto es importante hacer mención a que el proyecto no afirma a la filosofía en su dimensión de sistema o cuerpo de conocimientos, sino más bien como práctica o experiencia que comprende los siguientes valores: el diálogo fundamentado como modo de producir conocimiento, la cooperación y la solidaridad como formas de trabajar con los otros miembros de la comunidad; la investigación constante y cuestionadora como manera de abordar los problemas sociales; la libertad para pensar lo aún no pensado, la igualdad como principio, la diferencia como condición de posibilidad de la existencia y como posibilidad de apertura para ser lo que todavía no somos.

De acuerdo con ello, podríamos decir que la filosofía que desplegamos en Caxias no es solo un ejercicio en general, sino que se trata de uno singular. Para nosotros la filosofía que experimentamos en Caxias es un caminar, un viajar que va de nosotros a nosotros mismos, con el objeto de ser siempre otros nosotros de los que iniciamos el viaje para volver a comenzar. La filosofía como viaje y los viajes como filosofía constituyen una parte importante de nuestro proyecto, no solo porque nosotros, efectiva y materialmente 
viajamos mucho a distintas escuelas y universidades del mundo ${ }^{2}$, sino también porque los viajes forman parte de nuestro vivir y nuestro filosofar. Como sostiene Kohan (2016), refiriendose a Rodríguez: "Vive viajando, lo que significa que no vive para viajar sino que viaja para vivir" (p. 67).

En nuestro grupo hay una relación directa entre viaje y filosofía. Viaje y filosofía son ejercicios en los que lo interesante se encuentra en el proceso, en el transcurrir entre dos, tres, multiples puntos. De la misma forma que la filosofía, el viajar es estar en camino en un punto incierto o cierto, entre el inicio y el destino. El viajar es estar en un movimiento constante, cada paso es un ingreso en un nuevo infinito que nos abre a infinitas posibilidades. En este sentido el viajar no supone un despliegue paradójico, ni la inmobilidad de una aparente mobilidad. No se trata de la aporía de Zenón, sino de los múltiples infinitos de Cantor. Por ello pensamos que, sea en líneas rectas, circulares o risomáticas, el viaje siempre nos abre a lo infinitamente divisible, a un inagotable recorrido del movimiento que va como, hemos dicho, de nosotros a un nostros mismos distinto.

Para ilustrar esta idea nos apoyamos en Anábasis de Jenofonte (1999). En dicho texto, el autor narra el viaje de diez mil mercenarios griegos por la actual Turquía en busca de su hogar. Ciro el Joven convoca de forma paga a los griegos para participar en las guerras civiles para derrocar a su hermano Artajerjes. Tras la muerte de Ciro el Joven en la batalla de Cunaxa, los mercenarios deciden emprender el viaje de vuelta a casa. Los diez mil, perdidos, abandonados a su suerte y desorientados, inician su viaje hacia lo que ellos consideran su hogar. Se trata del inicio de un partir orientado en el que los mercenarios se proponen volver a partir del propio extravío. En este deambular por Siria, Babilonia, Turquía y Armenia, hasta llegar a las costas del Mar Negro, los guerreros se transforman en viajantes, en errantes en busca del hogar. Hay una transformación en la que está presente aquello de lo que los mercenarios son capaces, de guerrear -a lo largo del texto se detallan innumerables enfrentamientos-, pero también aquello que los invita a ir más allá de lo que saben hacer, construir e instalar. Esto puede verse la famosa expresión de júblio que los griegos lanzan al ver el mar: "iEl mar!, iEl mar!,

2 Los integrantes del NEFI han viajado como parte de su formación a países tales como: Portugal, Argentina, Grecia, Chile, Venezuela, México, Italia, Estados Unidos y Suecia, entre otros. 
iEl mar!" (Jenofonte, iv,7, 21-27). Los guerreros, ahora viajantes, ante la proximidad del hogar lanzan expresiones de júbilo que los coloca ante una nueva transformación. Para continuar el viaje que nos lleva al hogar será necesario ser marino. Es la invitación, el recuerdo a ser lo que eran en un inicio, pero de forma diferente. Esta transformación es indefinida mientras se está en viaje, ya que viajar es transformarse constantemente.

De esta manera el viaje, el retorno de Anábasis es, para nosotros, una metáfora de las implicancias del hacer filosofía. En viaje estamos en casa, en transformación permanente como en la escuela. Por ello decimos que Caxias es nuestro viaje, nuestra casa y nuestra escuela. Este viaje encuentra inspiración en una serie de pensadores entre los que podemos mencionar no solo a Rodríguez, sino también al Sub Comandante Marcos, Sócrates, Deleuze, Foucault y Freire entre otros muchos. En este trabajo nos hemos centrado en un concepto del maestro caraqueño que nos parece interesante para pensar nuestra práctica en Caxias.

Entre los elementos que rescatamos de Rodríguez, nos interesa su forma de entender la ciudadanía y el viajar. Respecto de la ciudadanía pensamos que la potencia de este concepto no se basa exclusivamente en el votar o conocer nuestros derechos y obligaciones. Estamos convencidos de que estos contenidos deben formar parte delcurrículo de toda escuela. Lo que nos interesa destacar en este trabajo es que nos resulta muy interesante explorar y desplegar las posibilidades para una vida igualitaria ofrecida a todos. Se trata de un pensar que valora el pensamiento de cada uno en su singularidad como medio de construcción novedoso de nuestra vida en conjunto. Allí comprendemos que lo público es de todos. Afirmamos la necesidad de pensar una cotidianidad, una escuela y una vida apoyada en el principio de igualdad. Sobre ella y a partir de ella nos relacionamos filosóficamente niños, niñas y adultos, discutiendo con la miríada de singularidades que nos constituyen.

Por último, el viajar es un elemento central de la vida del maestro. Desde su nacimiento como expósito en 1769, su vida y su pensamiento se encuentran marcados por el viajar. España, Francia, Austria, Alemania y supuestamente Rusia, Jamaica y Estados Unidos, constituyen un itinerario inicial en el que Rodríguez vive, piensa y educa. Luego Colombia, Ecuador, Perú, Bolivia y Chile ven pasar su vida y su escritura. Este caminar, no es un viajar de 
turista, ni un peregrinar, ni una marcha, es una afirmación de un modo de vida que se transforma permanentemente. El maestro constituye su práxis política y teórica en este andar trashumante. En cada ciudad, pueblo o villorrio del continente americano Rodríguez despliega sus ideas a favor de una escuela distinta a las existentes. Principio igualitario, escuela, vida cuidadosa y atenta se desprenden del andar errante de Rodríguez contra la escolarización jerárquica, segmentada y enciclopédica de las escuelas tradicionales. Siempre los mismos conceptos repetidos hasta el cansancio, pero de forma diferente. Toda su obra es el viaje trazado por el convencimiento de principios pensados y repensados permanentemente. Chuquisaca, Concepción y Latacunga son tan solo algunos ejemplos de lo que decimos. En esas ciudades el maestro abrió escuelas iguales, pero diferentes en cada caso. Más allá de las singularidades propias de cada contexto, la diferencia en la igualdad que sostenemos se refiere a los conceptos que allí se despliegan. El principio igualitario que se afirma en ellas es idéntico, no obstante, también es diferente. Entre Chuquisaca y Latacunga el maestro ha viajado por Bolivia, Chile, Perú y finalmente Ecuador. Su derrotero le permite pensar este principio a la luz de los contrastes internos de las sociedades poscoloniales. Es consciente de la utilización fragmentada de este concepto en boca de los patriotas. Por todos lados escucha y lee a intelectuales y políticos hablar de igualdad. Todos ellos llenan páginas de libros y decretos alabando la igualdad como condición de posibilidad del sistema republicano. Sin embargo, cuando se trata de llevar a la práctica este principio, la dura realidad muestra que en América hay algunas personas más iguales que otras. Tras las guerras de emancipación, la realidad política y social de nuestro continente cambia muy poco en relación con el periodo colonial. El sistema de castas se sostiene implicitamente en nuestro continente de forma solapada. Los sectores acomodados no tienen la mínima intención de revisar esta situación y Rodríguez lo sabe. Su vida y sus viajes constituyen una manifestación militante contra este estado de cosas. Por ello, el concepto de igualdad asume un ámbito central de su vida, su lucha y su transitar docente. La igualdad que afirma en Chuquisaca y en Latacunga es la misma -axiomática, colectiva y para todos y cualquiera-, pero también es diferente. En Latacunga hace mención explícita a la igualdad entre las castas. Los pueblos originarios son explícitamente incluidos en el alcance de este principio igualitario. En Consejos de amigo dado al Colegio de 
Latacunga (Rodríguez, 1999b) menciona la importancia del quechua y la preferencia de esta lengua sobre el latín para la consolidación de sociedades republicanas. En este texto hay referencias explícitas a la dignidad e importancia de los pueblos originarios, que no están presentes en otros trabajos suyos. En dicho trabajo exclama enfáticamente: "¿iEs posible!? Que vivamos con los Indios, sin entenderlos? [...] Ellos hablan bien su Lengua, i nosotros ni la de ellos ni la nuestra" (Rodríguez, 1999b, p. 37).

Esta afirmación muestra la reflexión del maestro sobre el problema complejo de la realidad social americana tras las guerras de emancipación. Allí Rodríguez asume explícitamente el problema y le pone nombre. Los iguales son todos los ciudadanos de las nuevas repúblicas, incluso los indios. Como tal, merecen el mismo respeto y dignidad por parte del Estado que el resto de los ciudadanos.

En otros textos el tema se encuentra diluido en el principio igualitario que se propone desplegar. En Sociedades Americanas sostiene "escuela para todos, porque todos son ciudadanos" (Rodríguez, 1999a, p. 284). En este texto, anterior al trabajo de Latacunga, se afirma la igualdad con carácter axiomático, pero sin referencia explícita a las castas.

Para nosotros, la explicitación de un grupo en particular es un ejemplo de la necesidad de Rodríguez, años después, de aclarar qué debe entenderse por todos en una sociedad republicana. Podría decirse que ambos textos afirman un mismo concepto: la igualdad, pero lo hacen de una forma distinta. Mientras que el primero se refiere al concepto en cuestión de una forma general y abstracta, el segundo se centra en la singularidad de un grupo en particular. Establecer los motivos que llevaron a Rodríguez a realizar esta explicitación exceden los límites de nuestro trabajo. No obstante, pensamos que dicha diferencia bien puede obedecer a las particularidades y singularidades que Rodríguez observa en distintos contextos que recorre en sus viajes. Según nuestro punto de vista, el andar rodrigueano es el ámbito en el que piensa y repiensa constantemente sus conceptos.

Por ello decimos que, de la misma manera que Anábasis, el viajar es un retorno permanente a sí mismo pero diferente de sí mismo. En Caxias sostenemos y vivimos esta idea de viaje, presente en Rodríguez como filosofía. Como sostiene Kohan (2016), 
refiriéndose a Rodríguez, pensamos que de viaje nos sentimos "de camino hacia un nuevo proyecto, hacia un nuevo inicio, hacia una nueva vida" (p. 67).

\section{Conclusión}

De acuerdo con lo escrito, el proyecto del NEFI se inspira en el pensamiento de filósofos y filósofas, entre los cuales se encuentra Simón Rodríguez. En lo referente a él, hemos intentado mostrar cómo pensamos los conceptos de ciudadano y viaje para potenciar nuestras filosofías. La ciudadanía es concebida como una apuesta anclada en el despliegue de la afirmación igualitaria. Los profesores se sienten capaces de hablar por ellos mismos sobre un concepto que se encuentra preconcebido y sometido a un conjunto de normas y principios pensados por otros y para otros. En Caxias se asume la apuesta rodrigueana de intentar nuevas formas de pensar la igualdad y la ciudadanía. Parafraseando al maestro caraqueño, podríamos decir que el proyecto se mueve y se desarrolla en la disyuntiva entre la invención de nuevos modos de intervenir una lógica reproductora del statu quo o errar legitimándolo. De acuerdo con ello, la ciudadanía que afirmamos en Caxias es uno de esos modos diferentes de habitar el mundo. Inspirados en la idea de igualdad de Rodríguez, sostenemos que la ciudadanía no es algo que debe ser formado. Para nosotros, se trata del despliegue en una práctica igualitaria, reflexiva y potente de todas y todas para todos y todas.

Por todo lo anterior, la escuela popular de Rodríguez es para nosotros una inspiración. "Inventamos o erramos" es una frase que materializa nuestra idea de filosofar, caminar y vivir. El proyecto se constituye y recrea permanentemente en su despliegue cotidiano con los niños, niñas, jóvenes y adultos que participamos de los diversos y múltiples encuentros que se generan a lo largo de los años. Allí no hay un orden para imitar ni un modelo que copiar. Se trata de un transitar, de un viajar, de un pensar y de un vivir que genere las condiciones para un nuevo transitar, viajar, pensar y vivir: la filosofía como vida y como viaje de transformación. La filosofía en Caxias sí encaja. 


\section{Referencias}

Aquino de, T. (1947). Suma Teológica, (Edición bilingüe con el texto crítico de la Leonina), (Vol. 16) Madrid: BAC.

Cansanello, O. (2008). Ciudadano / Vecino. En N. Goldman(Ed.), Lenguaje y revolución: Conceptos políticos claves en el Río de la plata, 1780 -1850 (19-34). Buenos Aires: Prometeo.

Deleuze, G., \& Foucault, M. (2006). Os intelectuais e o poder. En G. Deleuze, A ilha deserta e outros textos (pp. 265-273). São Paulo: Iluminuras.

Donghi, T. (2010). Tradición política española e ideología revolucionaria de Mayo. Buenos Aires: Prometeo

Durán, M. (2010). El concepto de ciudadano en el pensamiento de Simón Rodríguez: igualdad y universalidad. Estudios de filosofía práctica e historia de las ideas, 12(1), 9-21.

Guerra, F. (1999). El soberano y su reino. Reflexiones sobre la génesis del ciudadano en América Latina. En H. Sábato (Coord.), Ciudadanía política y formación de las naciones. Perspectivas históricas de América Latina ( pp. 33-61). México: FCE, Colegio de México.

Jenofonte. (1999). Anábasis. Madrid: Cátedra.

Kohan, W. (2016). El maestro Inventor. Simón Rodríguez. Caracas: Ediciones del Solar.

Núñez, F. (2007). El concepto de vecino/ciudadano en Perú (17501850). Araucaria Revista Iberoamericana de Filosofía, Política y Humanidades, 9(17), 235-253.

Quijada, M. (2008). Una constitución singular. La carta gadatina en perspectiva comparada. Revista de Indias, 68(242), 15-38.

Rodríguez, S. (1999a). Obras Completas, Tomo I. Caracas: Presidencia de la República.

Rodríguez, S. (1999b). Obras Completas, Tomo II. Caracas: Presidencia de la República. 